\title{
HIERARCHICAL ALGORITHM IN DTM GENERATION AND AUTOMATIC EXTRACTION OF ROAD FROM LIDAR DATA
}

\author{
Li Hui-ying ${ }^{\text {a }}$, Xu Yu-jun ${ }^{\text {a }}$, Wang Zhi ${ }^{\text {b }}$, Lu Yi-nan ${ }^{\text {a* }}$ \\ ${ }^{a}$ College of Computer Science and Technology, Jilin University, Qianjin Str.2699, Changchun City, 130012, China \\ - lihuiying@jlu.edu.cn \\ ${ }^{\mathrm{b}}$ College of Resources and Civil Engineering, Northeastern University, Shenyang, 110819,China - \\ wangzhi@mail.neu.edu.cn
}

\section{KEY WORDS: LIDAR, DTM, Road Extraction}

\begin{abstract}
:
Growing demand for an efficient land use above and below the ground is motivating cadastre and land management systems to move from traditional 2D systems toward three dimensional ones. Airborne laser technology offers direct acquisition of dense and accurate 3D data. In order to get 3D road this paper proposes a hierarchical algorithm to extract terrain point from LIDAR data. We stratify the raw LiDAR data according to the height, judge terrain points and non-terrain points by the connectivity. In the case of road network, it indicates the morphological characteristics of network structure with a certain length continuous strip and small difference in intensity. All these information, including elevation information, the intensity information, the morphological characteristics and other local features, are used for extracting the road network from DTM. Local morphological filtering method is implementing for finding clear boundaries and rich details of the road profile. Following the presentation of the algorithm results for this approach are shown and evaluated
\end{abstract}

\section{INTRODUCTION}

Using the LiDAR(Light Detection And Ranging) technology, we can obtain the 3-D information of the earth surface quickly and accurately[1]. By contrast with traditional photogrammetry, the 3D urban data capturing using LiDAR is of higher speed, higher vertical accuracy and lower cost [2]. Road extraction from remotely sensed data is a challenging issue and has been approached in many different ways by Photogrammetric and digital image processors. Some of the methods are quite complex and require the fusion of several data sources or different scale space images. Most of the existing road extraction algorithms use the method of clustering or growth. Such as Jeong-Heon Song makes an assessment of possibility with intensity data to classify the point cloud[3], and Farhad Samadzadegan use classifier fusion method [5]. Liang Gong uses K-means clustering algorithm to detect roads based on intensity return and the 3D information of LiDAR data [1]. But these methods only consider the intensity value of LiDAR data. In practice, the classification methods, which only use the intensity values, are difficult to achieve accurate classification results. The clustering methods often require pre-machine learning and the different regions have different roads characteristics. Therefore, the clustering methods have difficulties in producing accurate extraction results of different types of roads. Thus the clustering methods do not have broad applicability. In the case of region growing approach, it is not only has a great dependence on the seeds, but also high time complexity. This paper describes a new method of the automatic DTM generation and road extraction and tries to overcome the lack of methods mentioned above.

\section{HIERARCHICAL ALGORITHM}

Generalization DTM from LiDAR data was often used to extract 3D road. It computes the points cloud to access DTM, then sets a certain threshold, and let point in point cloud subtract DTM , if the value is greater than threshold then delete it. This method cost high time complexity. This article will use a hierarchical algorithm to divide the original data according to the height. Through the judgement of empty holes produced by stratification, rule out points unground.

For the purpose of this paper, we define LIDAR data point as pi,

$$
p_{i}=(l p x, l p y, l p z, l p i)
$$

where $I p x$, Ipy, and $l p z$ represent the last pulse laser strike 3D coordinates and Ipi represents the intensity of the point. Let $S$ represents the set of all laser points,

$$
S=\left\{p_{1}, p_{2}, \ldots \ldots p_{n}\right\}
$$

The creation of the layer is defined by (3).

$$
S z_{j}=\left\{p_{i} \in S: j \times \Delta t<p_{i l p z} \leq(j+1) \times \Delta t\right\}
$$

where $S z_{j}$ denotes the j-th layer, $p_{i l p z}$ is the last pulse $\mathrm{z}$ coordinate of $p_{i}, j$ is the number of the layer and $\Delta t$ is the thickness of the layer. Then we get these layers as shown in Figure 1.

Due to the change trend of the ground is slow and connect to each other, it produces a large slice connected area (shown in Figure 1) in a horizontal section. The large connection areas are ground.

\footnotetext{
* Corresponding author. Lu Yi-nan ; Tel: +8613504330645;Email:luyn@jlu.edu.cn
} 


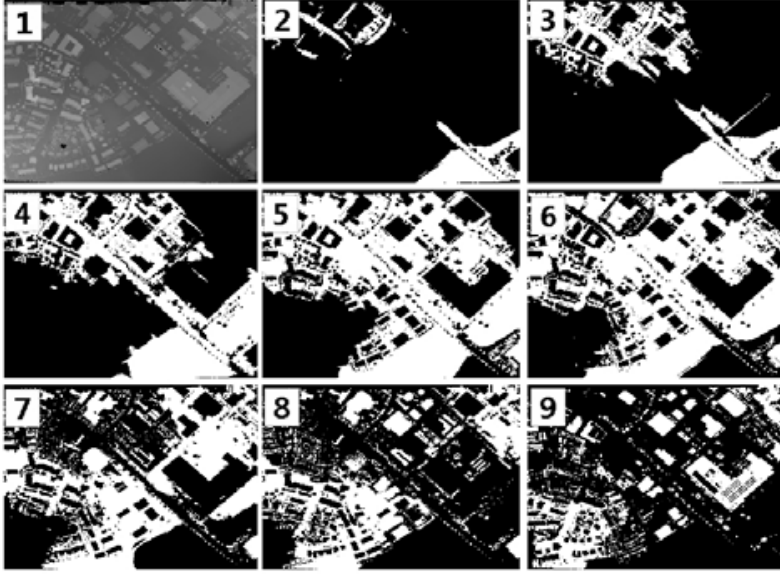

Figure 1. the first is elevation map; 2-9 are layers with different height.

There are some empty holes in each connection areas $S z_{j}$ (shown in Figure 1). One kind is that building's or vegetation's "top" and ground in space is not connecting to each other. These empty holes of the corresponding position can be eliminated. The second kind of empty holes are caused by the terrain. In the large connected region some small higher areas may be exist. These areas will produce empty holes and these empty holes should be retained. The difference between the tow kinds empty holes is showed in the figure 2.

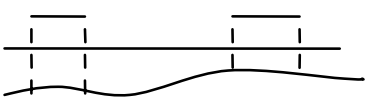

(a)

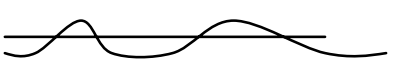

(b)
Figure 2. (a) The empty holes caused by building or vegetation. (b) The empty hole caused by small higher area.

In each layer there are some of the lesser connection areas which are caused by two reasons. One is the height diffence between buildings (vegetation) and ground. The other is the local small low-lying ground.

First of all we clear the noise of the original data. Then the data is stratified according to height. Value of layer-thickness should not be too large, otherwise accuracy is low. This paper choose 1 meter as a layer-thickness. And then began to merge gradually from the lowest layer, making judgment of the layer connection areas in the process of the merger .If there are empty holes, making judgment, delete empty holes caused by building and vegetation. As shown in Figure 3, we get these terrain points.

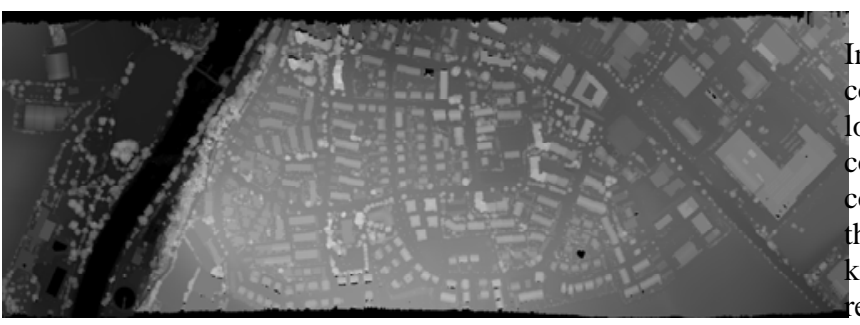

n order to get more accurate results and reduce the complexity, this article deals with a smaller local scope. In the ocal scope increase intensity value step by step, and judge connection areas within the local region. When these connected areas are greater than a certain threshold, judge hose shape and topological position respectively for deciding keep or not. And then make judgment in next local small egion. Finally we get rough road profile.

Figure 3. (a) LiDAR data 


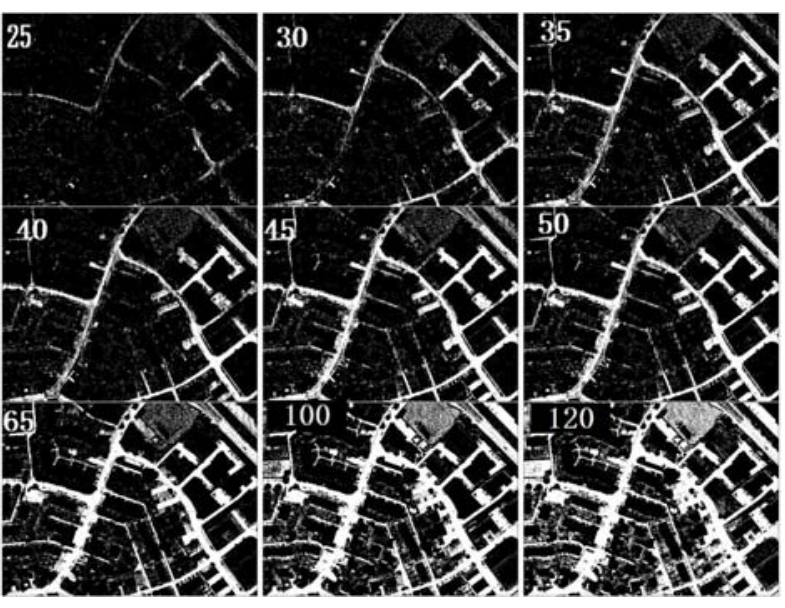

Figure 4. Comparison of different intensity

For these areas with the shape are similar circle or square, we adopt the ratio of the circumference and area as judgment standard. For square, $a$ and $b$ are defined as side, $S$ represents area and $W$ represents circumference.

$$
\frac{S}{W}=\frac{a b}{2(a+b)}
$$

Because the road is strip shape, so we suppose $\mathrm{a}>\mathrm{b}$ and define $m=b / a$

$$
\frac{S}{W}=\frac{m a^{2}}{2 a(1+m)}=\frac{m a}{2(1+m)}=\frac{\sqrt{m S}}{2(1+m)}
$$

So the area and perimeter of the road meet the following conditions:

$$
\frac{S}{W}<\frac{\sqrt{m S}}{2(1+m)} \quad(\mathrm{m}<1)
$$

From the equation we can see, in case of certain area, the smaller of $m$ the greater of circumference.

This method can rule out circular area and rectangular area efficiently, but not good at other strange shape. So a Fourier descriptor is inducted to eliminate none-road areas.

Fourier descriptor is Fourier transform coefficient of object boundary curves. It is the frequency domain analysis results of object boundary curves signal. According to the properties of the Fourier transformation, Fourier descriptor relates to scale, direction of shape and curves starting points. Therefore it's necessary to normalize Fourier descriptor. Through translation, rotation and scale invariance, normalization Fourier descriptor can identify the shape of an object. In order to extract road accuracy, this paper employs simple training sets for Fourier descriptor.

Through the above constraints, the vast majority of the nonroad region has been eliminated as shown in figure 5 . Regions eliminated in the process are stored in addition which will be used in the following process.

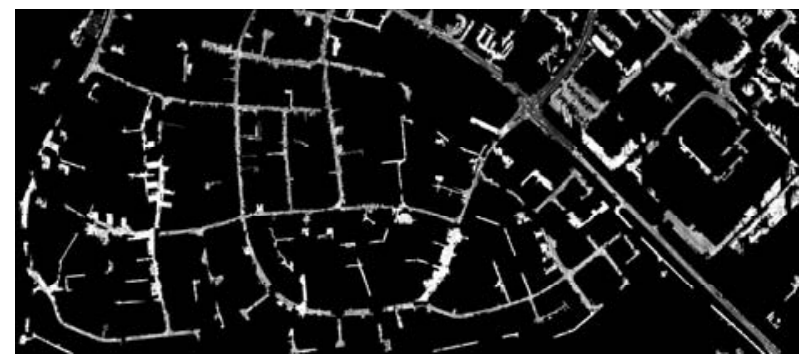

Figure 5. The main road frame

\subsection{Region Growing}

Most of the none-road area through the above steps has been excluded. This paper will put these not excluded points as seed, control and grow through some threshold .The road regional growth is also in the local, as data quantity is big, growing in the entire region will produce very high space complexity and time complexity. In order to be able to produce more accurate road, the paper will set the value very small, here set it 4 (in this paper the range of return intensity is $0-255$ ), the main consideration is a lot of points meet the conditions produced in the last step .As the threshold set is smaller, after growing will also have some breakpoints (mainly due to uneven distribution intensity of the road).This paper will take judgment methods of number of connection areas to make up breakpoint. The area removed in the previous step was preserved in another place, and put the area back to the road. If you join a "piece", the number of connection areas decrease, join the piece to road, or deleted it. The finally result is shown in the figure 6 .

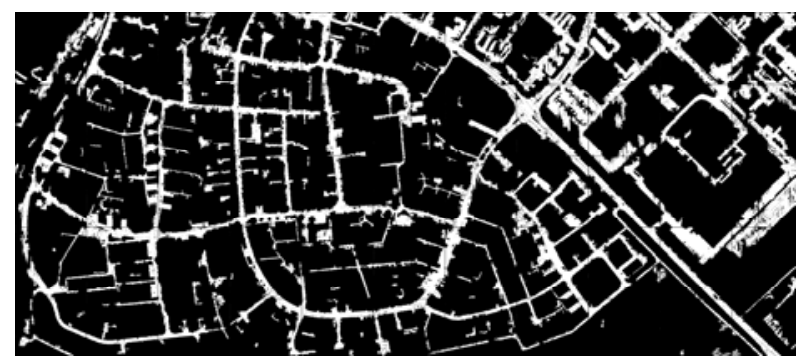

Figure 6. Road extraction result

\section{CONCLUSIONS}

In this study, hierarchical extraction algorithm for DTM generation is proved particularly suitable for the flat regions. For the fluctuant ground, we can increase the number of layers to achieve good results. Proposed hierarchical method of DTM extraction shows high accuracy and low complexity in the experiments. Local morphological filtering for road extraction can filtered out these areas which intensity values is close to the road, for example, the squares, parking lots and so on. Proposed methods are demonstrated of taking full advantage of low complexity, stability and widely applicability.

However, there are some issues that need further study:

1. In this article the geometry judgment effect is not very ideal, needs further study. 
2. For the road extraction, the thickness of the layer and layer's step size is very important. In order to get better result, we need a dynamic thickness and step size generation method.

\section{ACKNOWLEDGEWMENT}

Work described in this paper was funded by 973 Program (2011CB707102); National Natural Science Foundation of China (40901220, 41001302); Fok Ying Tong Education Foundation (122025).

\section{REFERENCES}

[1] Liang Gong, 2010.Automated Road Extraction from LiDAR Data Based on Intensity and Aerial Photo, Image and Signal Processing (CISP), 2010 3rd International Congress on, pp. 2130 - 2133

[2] Guofeng Wang, Yunling Zhang, 2011.3D Road Information Extraction from LiDAR Data Fused with Aerial-Images, Spatial Data Mining and Geographical Knowledge Services (ICSDM), 2011 IEEE International Conference on, pp. 362 - 366

[3] Jong-Suk Yoon, Jung-Il Shin, 2008.ASSESSING THE POSSIBILITY OF LAND-COVER CLASSIFICATION USING LIDAR INTENSITY DATA, Geoscience and Remote Sensing Letters, IEEE, Volume: 5 Issue:4,pp. 801 - 805

[4] Garland M., Heckbert P. S., 1997. Surface simplification using quadric error metrics, in: Proceedings of SIGGRAPH 1997, pp. 209-216.
[5] Samadzadegan F., Hahn M.,2009. Automatic Road Extraction from LIDAR Data based on Classifier Fusion, Urban Remote Sensing Event, 2009 Joint,pp.1-6

[6]Garland M., Zhou Y., 2005. Quadric-based simplification in any dimension, ACM Transactions on Graphics 24 (2), pp. 209-239.

[7]Lee C. H., Varshney A., Jacobs D., 2005. Mesh saliency, ACM Transactions on Graphics (Proceedings of SIGGRAPH 2005) 24, No. 3, pp. 659-666.

[8]Surazhsky V., Gotsman C. 2005. A qualitative comparison of some mesh simplification software packages. http://www.cs.technion.ac.il/ gotsman/AmendedPubl/Vitaly/ SimpStudy-final.pdf (Preprint).

[9]Wood, J. D., 1996. The geomorphological characterisation of digital elevation models $\mathrm{PhD}$ Thesis, University of Leicester, UK, pp. 185.

[10]Simon C., Peter K., Franz R., 2004. The Automatic Extraction of Roads from LIDAR Data, University of New South Wales, Australia, http://citeseerx.ist.psu.edu/viewdoc/download?doi=10.1.1.133 .520\&rep=rep1\&type=pdf.

[11]Farhad S.,Michael H.,behnaz B.,2009. Automatic Road Extraction from LIDAR Data basedon Classifier Fusion, Urban Remote Sensing Joint Event, http://ieeexplore.ieee.org/xpls/abs_all.jsp?arnumber=5137739 [12]Liang G., Yongsheng Z., Zhengguo L., 2010.Quanfu B. Automated Road Extraction from LiDAR Data Based on Intensity and Aerial Photo, International Congress on Image and Signal Processing 2010 3rd, pp.2130-2133. 